05.3

\title{
Влияние атомов замещения и внедрения на магнитные свойства соединения $\mathrm{Sm}_{2} \mathrm{Fe}_{17}$
}

\author{
(C) С.В. Веселова ${ }^{1}$, И.С. Терёшина ${ }^{1}$, В.Н. Вербецкий ${ }^{1}$, К.В. Захаров ${ }^{1}$, В.Б. Тверской ${ }^{1}$, А.Н. Васильев ${ }^{1,2}$ \\ ${ }^{1}$ Московский государственный университет им. М.В. Ломоносова, Москва, Россия \\ ${ }^{2}$ Южно-Уральский государственный университет, Челябинск, Россия \\ E-mail: sv_veselova@mail.ru
}

Поступило в Редакцию 10 февраля 2021 г.

В окончательной редакции 15 марта 2021 г.

Принято к публикации 16 марта 2021 г.

Методом индукционной плавки в инертной атмосфере получены сплавы $\left(\mathrm{Sm}_{1-x} R_{x}\right)_{2} \mathrm{Fe}_{17}(R=\mathrm{Ho}, \mathrm{Er}$; $x=0.1,0.2,0.4)$. Исследованы структура и магнитные гистерезисные свойства как исходных соединений, так и гидридов на их основе. Установлено, что гидриды $\left(\mathrm{Sm}_{1-x} R_{x}\right)_{2} \mathrm{Fe}_{17} \mathrm{H}_{y}$ сохраняют ромбоэдрический $\mathrm{Th}_{2} \mathrm{Zn}_{17}$ тип кристаллической структуры (пространственная группа $R \overline{3} m$ ) исходных соединений. Гидрирование приводит к значительному повышению температуры Кюри и намагниченности насыщения в области комнатной температуры. Определены составы с потенциально высокими магнитными характеристиками.

Ключевые слова: редкоземельные интерметаллиды, намагниченность, температура Кюри.

DOI: 10.21883/PJTF.2021.12.51058.18731

Интерметаллические соединения $\mathrm{Nd}_{2} \mathrm{Fe}_{14} \mathrm{~B}$ и $\mathrm{Sm}_{2} \mathrm{Fe}_{17}$ представляют особый интерес для исследователей как с фундаментальной, так и с практической точки зрения, поскольку на их основе осуществляется разработка высокоэффективных магнитотвердых материалов [1-3]. Известно, что частичная замена легкого редкоземельного металла (Р3М) Nd на тяжелые (Dy и Но) может приводить к значительному увеличению коэрцитивной силы магнитов на основе замещенных составов [4-6]. Известно также, что внедрение атомов азота в кристаллическую решетку $\mathrm{Sm}_{2} \mathrm{Fe}_{17}$ способствует возникновению гигантской одноосной магнитокристаллической анизотропии, которая превосходит анизотропию $\mathrm{Nd}_{2} \mathrm{Fe}_{14} \mathrm{~B}$ [7] и создает предпосылки для повышения коэрцитивной силы.

Цель настоящей работы состоит в поиске новых соединений на основе $\mathrm{Sm}_{2} \mathrm{Fe}_{17}$ с потенциально высокими характеристиками с помощью атомов замещения и внедрения. Поскольку влияние на магнитные свойства частичного замещения самария тербием и диспрозием подробно исследовалось ранее [8-10], нами произведено частичное замещение $\mathrm{Sm}$ атомами Но и $\mathrm{Er}$ и выполнен сравнительный анализ магнитных свойств всех полученных соединений типа $(\mathrm{Sm}, R)_{2} \mathrm{Fe}_{17}$. В качестве атомов внедрения был использован водород. Известно, что соединения $R_{2} \mathrm{Fe}_{17}$ способны абсорбировать значительное количество водорода (до 5 атомов водорода на формульную единицу), в то время как максимальное количество азота достигает только 3 атомов $\mathrm{N}$ на формульную единицу. Изменение магнитных свойств в двух системах $R_{2} \mathrm{Fe}_{17}-\mathrm{N}$ и $R_{2} \mathrm{Fe}_{17}-\mathrm{H}$ может быть достаточно сильным [10-14].

Образцы $\left(\mathrm{Sm}_{1-x} R_{x}\right)_{2} \mathrm{Fe}_{17} \quad(R=\mathrm{Ho}, \mathrm{Er} ; x=0.1,0.2$, $0.4)$ были приготовлены методом индукционной плавки из исходных компонентов высокой чистоты в инертной атмосфере. Сплавы подвергались гомогенизирующему отжигу в вакуумированных кварцевых ампулах при температуре $1273 \mathrm{~K}$ в течение $192 \mathrm{~h}$. Микроструктура и химический состав материалов в литом состоянии и после высокотемпературного отжига исследовались на сканирующем электронном микроскопе (СЭМ) „LEO EVO $50 \mathrm{XVP}^{\star}$ с приставкой EDAX. Рентгенографические исследования всех синтезированных образцов проводились на дифрактометре ДРОН-3М в монохроматизированном Со $K_{\alpha}$-излучении. Фазовый состав образцов исследовался с помощью ритвельд-анализа в программе RIETAN 2000. Гидрирование проводилось посредством прямой реакции предварительно механически измельченных исходных сплавов (навески массой до $15 \mathrm{~g}$ ) с водородом при температуре $473 \mathrm{~K}$ и давлении водорода $30 \mathrm{MPa} \mathrm{в} \mathrm{течение} \mathrm{3-5} \mathrm{h.} \mathrm{Синтез} \mathrm{гидридов} \mathrm{осуществлялся}$ на специальной установке, подробное описание которой можно найти в работе [15]. Исследования магнитных свойств были выполнены на вибрационном магнитометpe VSM-250 при комнатной температуре, а также с помощью измерительного комплекса PPMS-9 (Quantum Design) в широком диапазоне температур. Для определения температуры Кюри проводился термомагнитный анализ в области температур 290-800 K.

Для всех полученных образцов исследовалась их микроструктура. На рис. 1 для примера показана микроструктура сплава $\mathrm{Sm}_{1.6} \mathrm{Ho}_{0.4} \mathrm{Fe}_{17}$ до и после отжига. Установлено, что первоначально литой сплав состоит из трех фаз: основной фазы 2:17, фазы $(\mathrm{Sm}, \mathrm{Ho}) \mathrm{Fe}_{3}$ и $\alpha$-Fe (соответственно темно-серый, светло-серый и черный контрастный участки на рис. $1, a)$. После гомогенизирующего отжига исследуемый сплав состоит уже на 96 mass\% из основной фазы 2:17, кристаллизующейся в 


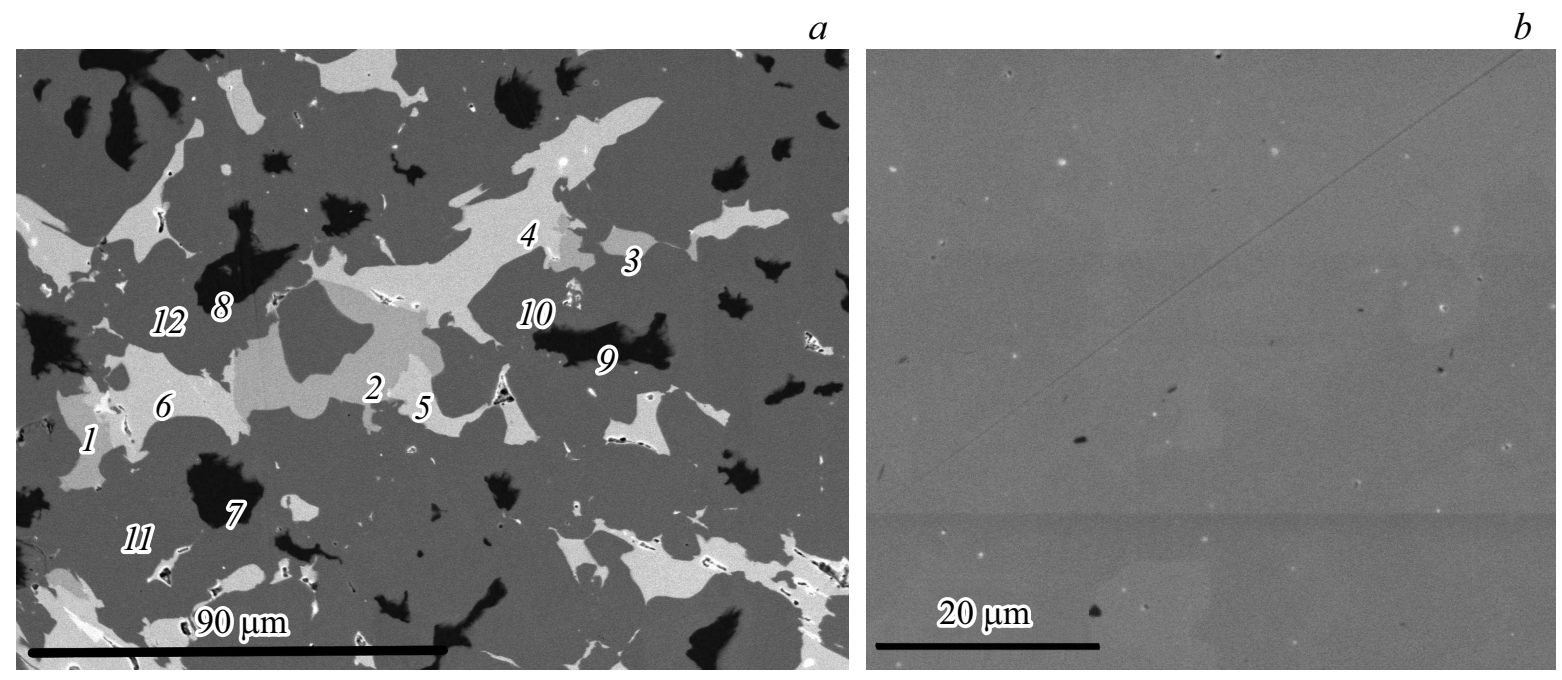

Рис. 1. СЭМ-изображения сплава $\mathrm{Sm}_{1.6} \mathrm{Ho}_{0.4} \mathrm{Fe}_{17} . a-$ в литом состоянии $\left(1-6-\right.$ фаза $(\mathrm{Sm}, \mathrm{Ho}) \mathrm{Fe}_{3}, 7-9-$ фаза $\alpha$-Fe, $10-12-$ ocновная фаза 2:17); $b$ - после высокотемпературной гомогенизации.

Структурные и магнитные характеристики соединений типа $\left(\mathrm{Sm}_{1-x}, R_{x}\right)_{2} \mathrm{Fe}_{17}(x=0,01,02,0.4)$ и их гидридов

\begin{tabular}{|c|c|c|c|c|c|c|c|c|}
\hline Образец & $a, \AA$ & $c, \AA$ & $c / a$ & $\mathrm{~V}, \AA^{3}$ & $\begin{array}{c}\Delta V / V_{0} \\
\%\end{array}$ & $\begin{array}{c}\sigma_{s}, \\
\mathrm{~A} \cdot \mathrm{m}^{2} / \mathrm{kg} \\
(300 \mathrm{~K})\end{array}$ & $\begin{array}{c}T_{C} \\
\mathrm{~K}\end{array}$ & $\begin{array}{c}\text { Лит. } \\
\text { ссылка }\end{array}$ \\
\hline $\mathrm{Sm}_{2} \mathrm{Fe}_{17}$ & 8.553 & 12.442 & 1.455 & - & - & 118 & 398 & {$[10]$} \\
\hline $\mathrm{Sm}_{2} \mathrm{Fe}_{17} \mathrm{H}_{2}$ & 8.653 & 12.506 & 1.445 & - & 2.9 & 149.6 & 554 & {$[10]$} \\
\hline $\mathrm{Sm}_{2} \mathrm{Fe}_{17}$ & 8.554 & 12.443 & 1.455 & 788.4 & - & - & 385 & {$[16]$} \\
\hline $\mathrm{Sm}_{2} \mathrm{Fe}_{17} \mathrm{H}_{4.7}$ & 8.682 & 12.550 & 1.446 & 819.2 & 3.9 & - & 565 & {$[16]$} \\
\hline $\mathrm{Sm}_{1.6} \mathrm{~Tb}_{0.4} \mathrm{Fe}_{17}$ & 8.551 & 12.446 & 1.456 & - & - & 96.5 & 388 & {$[8]$} \\
\hline $\mathrm{Sm}_{1.2} \mathrm{~Tb}_{0.8} \mathrm{Fe}_{17}$ & 8.539 & 12.442 & 1.457 & - & - & 90.3 & 390 & [8] \\
\hline $\mathrm{Sm}_{1.6} \mathrm{Dy}_{0.4} \mathrm{Fe}_{17}$ & 8.558 & 12.449 & 1.455 & - & - & 80 & 427 & [9] \\
\hline $\mathrm{Sm}_{1.2} \mathrm{Dy}_{0.8} \mathrm{Fe}_{17}$ & 8.543 & 12.402 & 1.452 & - & - & 77 & 429 & [9] \\
\hline $\mathrm{Sm}_{1.6} \mathrm{Ho}_{0.4} \mathrm{Fe}_{17}$ & $8.541(9)$ & $12.459(5)$ & 1.458 & 789.6 & - & $95(89)$ & 390 & Наст. раб. \\
\hline $\mathrm{Sm}_{1.6} \mathrm{Ho}_{0.4} \mathrm{Fe}_{17} \mathrm{H}_{4}$ & $8.649(7)$ & $12.526(2)$ & 1.448 & 815.2 & 3.2 & $127(123)$ & 510 & । \\
\hline $\mathrm{Sm}_{1.2} \mathrm{Ho}_{0.8} \mathrm{Fe}_{17}$ & $8.522(0)$ & $12.429(6)$ & 1.458 & 782 & - & $92(82)$ & 380 & $»$ \\
\hline $\mathrm{Sm}_{1.2} \mathrm{Ho}_{0.8} \mathrm{Fe}_{17} \mathrm{H}_{4.4}$ & $8.644(3)$ & $12.521(5)$ & 1.449 & 810.5 & 3.6 & $120(112)$ & 518 & $»$ \\
\hline $\mathrm{Sm}_{1.8} \mathrm{Er}_{0.2} \mathrm{Fe}_{17}$ & $8.555(8)$ & $12.453(9)$ & 1.456 & 788.8 & & $98(88)$ & 390 & $»$ \\
\hline $\mathrm{Sm}_{1.8} \mathrm{Er}_{0.2} \mathrm{Fe}_{17} \mathrm{H}_{4.4}$ & $8.632(1)$ & $12.511(7)$ & 1.449 & 815.6 & 3.3 & $123(119)$ & 525 & $»$ \\
\hline $\mathrm{Sm}_{1.6} \mathrm{Er}_{0.4} \mathrm{Fe}_{17}$ & 8.534 & 12.450 & 1.459 & - & - & 79 & 400 & {$[9]$} \\
\hline $\mathrm{Sm}_{1.2} \mathrm{Er}_{0.8} \mathrm{Fe}_{17}$ & 8.504 & 12.380 & 1.456 & - & - & 77 & 394 & [9] \\
\hline $\mathrm{Sm}_{1.2} \mathrm{Er}_{0.8} \mathrm{Fe}_{17}$ & $8.518(2)$ & $12.431(4)$ & 1.459 & 791.3 & - & $89(82)$ & 360 & Наст. раб. \\
\hline $\mathrm{Sm}_{1.2} \mathrm{Er}_{0.8} \mathrm{Fe}_{17} \mathrm{H}_{4.6}$ & $8.641(7)$ & $12.520(1)$ & 1.449 & 818.6 & 3.5 & $118(112)$ & 490 & $»$ \\
\hline
\end{tabular}

Пр име чан и е. В скобках для параметров элементарной ячейки $(a$ и $c)$ дана погрешность измерений, а для намагниченности $\sigma_{s}-$ уточненные для основной фазы (2:17) значения, рассчитанные по формуле (1). $\Delta V / V_{0}$ — относительное увеличение объема элементарной ячейки.

структурном типе $\mathrm{Th}_{2} \mathrm{Zn}_{17}$, что подтверждается рентгенофазовым анализом. Следы $\alpha$-Fe также присутствуют в образце.

В таблице представлены результаты рентгенографических исследований исходных образцов $\left(\mathrm{Sm}_{1-x} R_{x}\right)_{2} \mathrm{Fe}_{17}$ $(R=\mathrm{Ho}, \mathrm{Er} ; x=0.1,0.2,0.4)$ и их гидридов с максимальным содержанием в них водорода от 4 до 4.6 атомов Н на формульную единицу в сопоставлении с известными литературными данными $[8-10,16]$. Для гидридов замещенных соединений количество поглощен- ного водорода меньше, чем для гидридов $\mathrm{Sm}_{2} \mathrm{Fe}_{17} \mathrm{H}_{y}$ $\left(y_{\max }=5.2\right)$ [16]. Как видно из таблицы, все синтезированные гидриды сохраняют ромбоэдрический $\mathrm{Th}_{2} \mathrm{Zn}_{17}$-тип структуры, а увеличение объема кристаллической решетки гидрированных образцов составляет $3.2-3.9 \%$. Отметим, что при гидрировании происходит не только увеличение объема, но и характерное для гидридов уменьшение отношения параметров $c / a$.

Внедрение атомов водорода в кристаллическую решетку соединения $\left(\mathrm{Sm}_{1-x} R_{x}\right)_{2} \mathrm{Fe}_{17}$ оказывает влияние 
на их магнитные свойства. В таблице приведены значения температур Кюри (по данным термомагнитного анализа [17]), намагниченности насыщения $\left(\sigma_{s}\right)$ для всех исследуемых соединений с $R=$ Но и $\operatorname{Er}$ (полученные при комнатной температуре в полях напряженностью до $7 \mathrm{MA} / \mathrm{m})$, а также представлены известные литературные данные для других составов $[8-10,16]$. Анализ данных таблицы предоставляет важную информацию о совместном влиянии атомов замещения и водорода на магнитные свойства соединения $\mathrm{Sm}_{2} \mathrm{Fe}_{17}$. Видно, что при гидрировании $\left(\mathrm{Sm}_{1-x} R_{x}\right)_{2} \mathrm{Fe}_{17}(R=\mathrm{Tb}, \mathrm{Dy}, \mathrm{Ho}, \mathrm{Er}$; $x=0,0.1,0.2,0.4)$ удается значительно повысить температуру магнитного упорядочения и намагниченность насышения. Коэрцитивность по намагниченности $\left(j H_{c}\right)$ как для исходных образцов, так и для гидридов крайне мала. Наблюдается лишь незначительное ее увеличение для образцов после гидрирования (например, $j H_{c}=63$ и $96 \mathrm{Oe}$ для состава $\mathrm{Sm}_{1.6} \mathrm{Ho}_{0.4} \mathrm{Fe}_{17}$ и его гидрида соответственно). Полученные результаты находятся в полном согласии с данными работы по магнитным свойствам гидридов $\mathrm{Sm}_{2} \mathrm{Fe}_{17} \mathrm{H}_{y}(2 \leqslant y \leqslant 5.2)[16]$.

На рис. 2 представлены петли магнитного гистерезиса исходных образцов $\left(\mathrm{Sm}_{1-x} R_{x}\right)_{2} \mathrm{Fe}_{17} \quad(R=\mathrm{Ho}, \mathrm{Er}$; $x=0.1,0.2,0.4)$ и их гидридов с высоким содержанием водорода, измеренные при комнатной температуре. Частичная замена атомов самария атомами тяжелых РЗМ приводит к снижению намагниченности насыщения соединения $\mathrm{Sm}_{2} \mathrm{Fe}_{17}$ (см. таблицу) из-за того, что магнитные моменты атомов гольмия и эрбия упорядочиваются антипараллельно магнитным моментам атомов $\mathrm{Fe}$ и $\mathrm{Sm}$. После гидрирования намагниченность насыщения образцов значительно возрастает. Так, у $\mathrm{Sm}_{1.6} \mathrm{Ho}_{0.4} \mathrm{Fe}_{17} \quad \sigma_{s}=95 \mathrm{~A} \cdot \mathrm{m}^{2} / \mathrm{kg}$, в то время как $\mathrm{y}$ гидрида $\mathrm{Sm}_{1.6} \mathrm{Ho}_{0.4} \mathrm{Fe}_{17} \mathrm{H}_{4} \sigma_{s}=127 \mathrm{~A} \cdot \mathrm{m}^{2} / \mathrm{kg}$. С учетом пересчета намагниченности насыщения для содержания (в mass\%) фазы 2:17 в образце нами были также получены истинные значения $\sigma_{s}$ для магнитотвердой фазы с помощью формулы

$$
\begin{aligned}
\sigma_{s}(2: 17) & =\left[\sigma_{s}(\text { sample })-\sigma_{s}(\alpha-\mathrm{Fe})\right. \\
& \times \omega(\alpha-\mathrm{Fe})] / \omega(2: 17) \cdot 100,
\end{aligned}
$$

где $\sigma_{s}(\alpha-\mathrm{Fe})=210 \mathrm{~A} \cdot \mathrm{m}^{2} / \mathrm{kg}, \omega-$ содержание фазы в образце $[\mathrm{mass} \%]$. После пересчета значения $\sigma_{s}$ составляют $89 \mathrm{~A} \cdot \mathrm{m}^{2} / \mathrm{kg}$ для $\mathrm{Sm}_{1.6} \mathrm{Ho}_{0.4} \mathrm{Fe}_{17}$, а для образца $\mathrm{Sm}_{1.6} \mathrm{Ho}_{0.4} \mathrm{Fe}_{17} \mathrm{H}_{4}-123 \mathrm{~A} \cdot \mathrm{m}^{2} / \mathrm{kg}$. Уточненные данные также представлены в таблице.

Поскольку гидрид $\mathrm{Sm}_{1.6} \mathrm{Ho}_{0.4} \mathrm{Fe}_{17} \mathrm{H}_{4}$ демонстрировал наилучшие магнитные характеристики среди исследуемых нами составов, для него были проведены магнитные измерения в широком интервале температур 4.2-390 K в полях напряженностью до $7 \mathrm{MA} / \mathrm{m}$ (рис. 3). Установлено, что в данном интервале температур намагниченность насыщения изменяется довольно слабо, что свидетельствует о высокой термостабильности полученного образца благодаря значительному повышению температуры Кюри (на $120 \mathrm{~K})$.

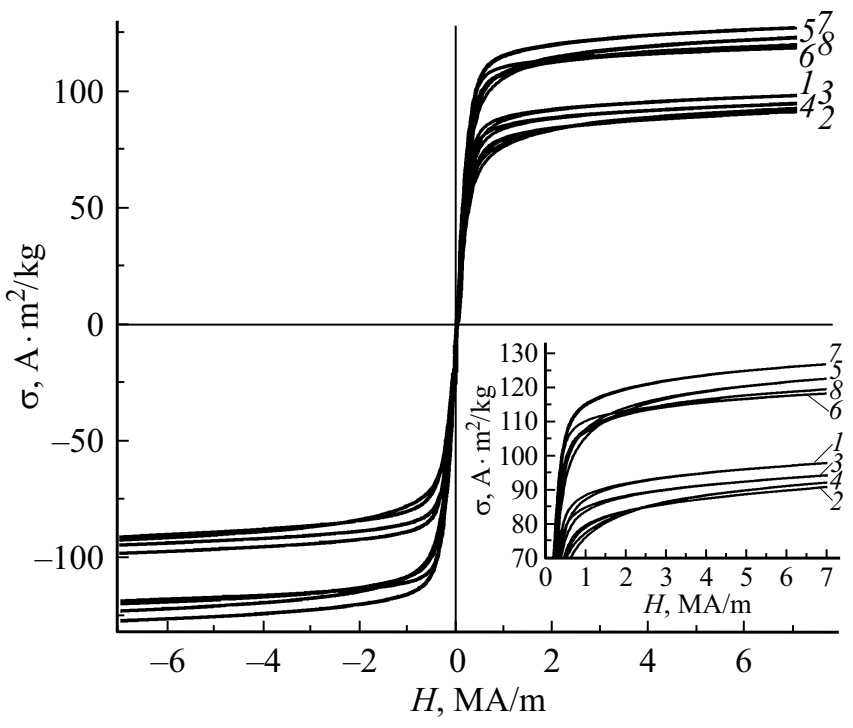

Рис. 2. Петли магнитного гистерезиса исходных образцов $\mathrm{Sm}_{1.8} \mathrm{Er}_{0.2} \mathrm{Fe}_{17}$ (1), $\mathrm{Sm}_{1.2} \mathrm{Er}_{0.8} \mathrm{Fe}_{17}$ (2), $\mathrm{Sm}_{1.6} \mathrm{Ho}_{0.4} \mathrm{Fe}_{17}$ (3), $\mathrm{Sm}_{1.2} \mathrm{Ho}_{0.8} \mathrm{Fe}_{17}$ (4) и их гидридов $\mathrm{Sm}_{1.8} \mathrm{Er}_{0.2} \mathrm{Fe}_{17} \mathrm{H}_{4.4}$ (5), $\mathrm{Sm}_{1.2} \mathrm{Er}_{0.8} \mathrm{Fe}_{17} \mathrm{H}_{4.6}$ (6), $\mathrm{Sm}_{1.6} \mathrm{Ho}_{0.4} \mathrm{Fe}_{17} \mathrm{H}_{4}$ (7), $\mathrm{Sm}_{1.2} \mathrm{Ho}_{0.8} \mathrm{Fe}_{17} \mathrm{H}_{4.4}$ $(8)$, измеренные при комнатной температуре. На вставке фрагменты петель в увеличенном виде.

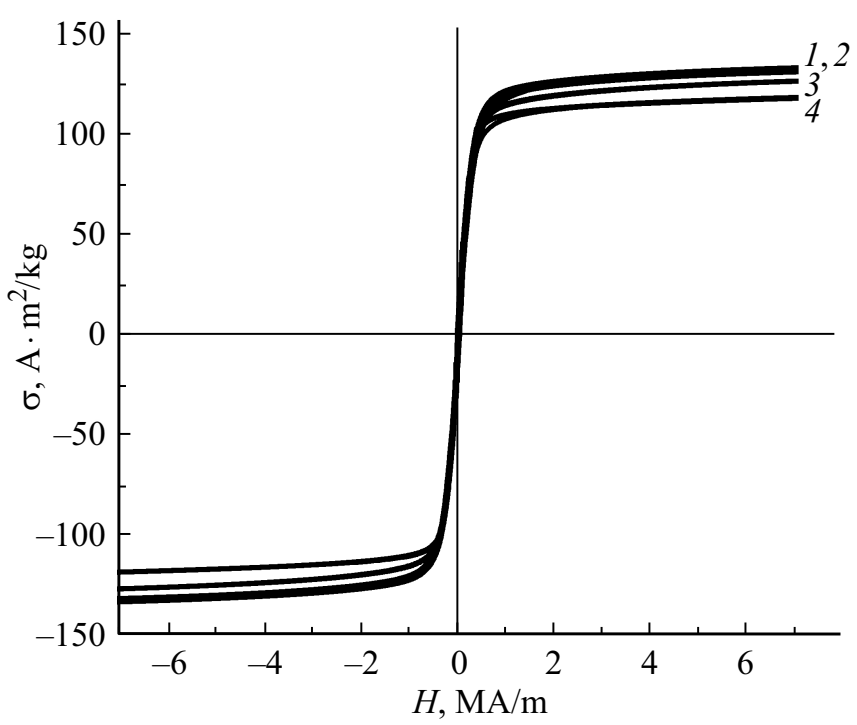

Рис. 3. Петли магнитного гистерезиса гидрида $\mathrm{Sm}_{1.6} \mathrm{Ho}_{0.4} \mathrm{Fe}_{17} \mathrm{H}_{4}$, измеренные при различных температурах: $4.2(1), 77(2), 300$ (3) и $390 \mathrm{~K}(4)$.

В заключение отметим, что частичное замещение атомов самария атомами тяжелых РЗМ приводит к небольшому снижению абсорбционной способности замещенных соединений по сравнению с $\mathrm{Sm}_{2} \mathrm{Fe}_{17}$, а также оказывает колоссальное влияние на основные магнитные свойства. Фундаментальные характеристики, полученные для гидридов, важны для разработки новых магнитных материалов с заданным комплексом свойств, способных эксплуатироваться в водородсодержащих средах. 


\section{Финансирование работы}

И.С. Терёшина благодарит Российский научный фонд за финансовую поддержку работы (проект № 18-1300135). А.Н. Васильев благодарит Правительство РФ за финансовую поддержку работы (акт 211, контракт № 02.A03.21.0011).

\section{Конфликт интересов}

Авторы заявляют, что у них нет конфликта интересов.

\section{Список литературы}

[1] J.M.D. Coey, Magnetism and magnetic materials (Cambridge University Press, 2010). https://doi.org/10.1017/CBO9780511845000

[2] H. Kronmüller, S. Parkin, in: Handbook of magnetism and advanced magnetic materials (Wiley, N.Y., 2007).

[3] O. Gutfleisch, M.A. Willard, E. Brück, C.H. Chen, S.G. Sankar, J.P. Liu, Adv. Mater., 23 (7), 821 (2011). https://doi.org/10.1002/adma.201002180

[4] H. Ucar, D.S. Parker, I.C. Nlebedim, R.W. McCallum, S.K. McCall, M.P. Paranthaman, Adv. Mater. Res., 4 (4), 227 (2015). https://doi.org/10.12989/amr.2015.4.4.227

[5] N.V. Kostyuchenko, I.S. Tereshina, D.I. Gorbunov, E.A. Tereshina-Chitrova, K. Rogacki, A.V. Andreev, M. Doerr, G.A. Politova, A.K. Zvezdin, Intermetallics, 124, 106840 (2020). DOI: 10.1016/j.intermet.2020.106840

[6] I.S. Tereshina, N.V. Kudrevatykh, L.A. Ivanov, G.A. Politova, E.A. Tereshina, D. Gorbunov, M. Doerr, K. Rogacki, J. Mater. Eng. Perform., 26 (10), 4676 (2017).

DOI: $10.1007 / \mathrm{s} 11665-017-2952-8$

[7] H.Fujii, H. Sun, in Handbook of magnetic materials, ed. by K.H.J. Buschow (North Holland, Amsterdam, 1995), vol. 9, p. 303-404. DOI: 10.1016/S1567-2719(05)80007-1

[8] M.Q. Huang, Y. Zheng, K. Miller, J.M. Elbicki, S.G. Sankar, W.E. Wallace, J. Appl. Phys., 70 (10), 6024 (1991). DOI: $10.1063 / 1.350081$

[9] O. Tegus, Y. Lu, N. Tang, J.X. Wu, M. Yu, Q.A. Li, R.W. Zhao, Y. Jian, F. Yang, IEEE Trans. Magn., 28 (5), 2581 (1992). DOI: 10.1109/INTMAG.1992.696380

[10] X. Rengen, W. Xinhua, W. Jianmin, P. Hongge, C. Changpin, W. Qidong, D. Lichi, J. Trans. Nonferrous Met. Soc. China, 9 (1), 40 (1999).

[11] I.S. Tereshina, S.A. Nikitin, K.P. Skokov, T. Palewski, V.V. Zubenko, I.V. Telegina, V.N. Verbetsky, A.A. Salamova, J. Alloys Compd., 350 (1-2), 264 (2003). DOI: $10.1016 / \mathrm{S} 0925-8388(02) 00957-\mathrm{X}$

[12] E.A. Tereshina, A.V. Andreev, Intermetallics, 18 (6), 1205 (2010). DOI: 10.1016/j.intermet.2010.03.008

[13] S. Nikitin, W. Suski, I. Tereshina, J. Stepien-Damm, W. Iwasieczko, H. Drulis, K. Skokov, J. Alloys Compd., 404-406, 172 (2005). https://doi.org/10.1016/j.jallcom.2005.03.101

[14] S. Nikitin, I. Tereshina, E. Tereshina, W. Suski, H. Drulis, J. Alloys Compd., 451 (1-2), 477 (2008).

DOI: $10.1016 /$ j.jallcom.2007.04.106

[15] С.В. Веселова, В.Н. Вербецкий, А.Г. Савченко, И.В. Щетинин, Материаловедение, № 4, 8 (2018).
[16] O. Isnard, S. Miraglia, J.L. Soubeyroux, D. Fruchart, P. l'Héritier, J. Magn. Magn. Mater., 137 (1-2), 151 (1994). DOI: 10.1016/0304-8853(94)90201-1

[17] S.V. Veselova, M.A. Paukov, I.S. Tereshina, V.N. Verbetsky, K.V. Zakharov, D.I. Gorbunov, A.N. Vasil'ev, J. Rare Earths, in press. DOI: $10.1016 /$ j.jre.2020.08.010 